\title{
La motivación de los profesores del programa educativo de negocios durante el COVID-19
}

\author{
The teacher's motivation of the business development educational program during \\ COVID-19 \\ Ismael Acevedo-Sánchez ${ }^{a}$, Alfredo Castillo-Trejo ${ }^{b}$, Víctor H. Ortiz-Alcalá ${ }^{c}$, María L. Ortega- \\ Montiel $^{d}$
}

\begin{abstract}
:
The educational authorities have been concerned about the students in the confinement in relation to their motivation, in order to keep the classrooms open and with it their professional training, in this virtual way to we have had to adapt, nevertheless, little has been said about the teacher's motivation, this is an issue that should be paid attention to continue with this virtual education project, which circumstances have led us to. This research has been developed to a sample of 44 professors of the Business Development Educational Program of the Technological University of Tula-Tepeji. The teachers answered a questionnaire with 24 items, divided into 4 dimensions, that measure aspects such as; their perception of confinement due to the pandemic, their general digital skills before and during the pandemic, level of motivation, and their attitude towards their students in times of contingency. From result, it was possible to determine the level of motivation that these teachers experience in their daily school activities from confinement by Covid19.
\end{abstract}

\section{Keywords:}

Confinement, Covid-19, motivation, teachers

\section{Resumen:}

Durante el confinamiento las autoridades educativas se han preocupado particularmente de los alumnos con relación a su motivación para mantener las aulas abiertas y con ello su formación profesional, de esta forma virtual a la que nos hemos tenido que adaptar, sin embargo, poco se ha hablado de la motivación de los profesores y este, es un tema al que se debe prestar atención para seguir con este proyecto de educación virtual, a la que las circunstancias nos han orillado. Para ello se ha desarrollado esta investigación a una muestra de 44 profesores del Programa Educativo Desarrollo de Negocios de la Universidad Tecnológica de Tula-Tepeji, a quienes se les aplicó un cuestionario con 24 ítems, divididos en 4 dimensiones, que miden aspectos como; su percepción del confinamiento a raíz de la pandemia, sus competencias digitales generales antes y durante la pandemia, nivel de motivación, y la actitud de ellos para con sus alumnos en tiempos de contingencia; del resultado se pudo determinar el nivel de motivación que estos profesores experimentan en sus actividades escolares cotidianas a partir del confinamiento por el Covid-19.

\section{Palabras Clave:}

Confinamiento, Covid-19, motivación, profesores

\section{Introducción}

Desarrollar la motivación en los estudiantes para aprender, implica socializarlo con una disposición general, así como estimularlo situacionalmente en el proceso de implementación de lecciones y actividades de aprendizaje, es este sentido, el profesor como motivador, se encarga de valorar el aprendizaje de sus alumnos e

\footnotetext{
Autor de Correspondencia, Universidad Tecnológica de Tula-Tepeji, https://orcid.org/0000-0002-4167-3047, Email: ismael.acevedo@uttt.edu.mx

b Universidad Tecnológica de Tula-Tepeji, https://orcid.org/0000-0002-1063-497, Email: alfredo.castillo@uttt.edu.mx

c Universidad Autónoma del Estado de Hidalgo, https://orcid.org/0000-0003-3806-0060, Email: victor_ortiz@uaeh.edu.mx

${ }^{d}$ Universidad Tecnológica de Tula-Tepeji, https://orcid.org/0000-0003-0005-1935, Email: mariadelourdes.ortega@uttt.edu.mx
} 
impulsarlos a conseguir los objetivos planteados (Villafuerte, et. al., 2020).

De acuerdo a lo anterior el profesorado ha tenido que reinventarse en tiempo récord su forma de impartir clase y de relacionarse con los alumnos (Beltrán et. al., 2020), a fin de mantener el concepto de escuela abierta y seguir con la formación de los futuros profesionistas de este país.

No obstante, ese ímpetu por parte de los profesores, México corre el riesgo de un rezago educativo a consecuencia del Covid 19, derivado de la suspensión de clases presenciales, ocasionando una brecha de aprendizaje debido a que los estudiantes carecen de autorregulación y autodisciplina y sobre todo por la carencia de conexión a internet y computadoras (Schmelkes, 2020 citado por Cervantes 2020).

Esta situación ya era todo un gran reto en los modelos de educación tradicionales, y esto ha significado un doble reto en tiempos de pandemia. Los maestros influyen en la motivación de los estudiantes de muchas maneras; a través de interacciones diarias con ellos, en sus creencias sobre sus propias habilidades, en sus metas inmediatas y a largo plazo, en sus creencias sobre las causas de sus éxitos y fracasos, y en sus razones para desarrollar los trabajos académicos.

Pero que sucede si el profesor no se encuentra motivado, ¿de qué manera esto influye en los alumnos a su cargo?, para ello es necesario que las instituciones educativas centren su atención en analizar la motivación de los profesores, ya que de ellos dependerá la guía que tengan los estudiantes para poder sobrellevar esta etapa de confinamiento.

\section{DESARROLLO}

\section{MARCO TEÓRICO}

La motivación puede conceptualizarse como una combinación de procesos intelectuales, fisiológicos y psicológicos que decide, en un momento dado, con que vigor se actúa y en qué dirección se encauza la energía (Vidal y García, 2009), por otra parte, de acuerdo con Villafuerte et al., (2020) el profesor como motivador valora e impulsa a sus estudiantes a conseguir los objetivos planeados. "La motivación es el conjunto de factores internos que, con los estímulos externos de la situación, determinan la dirección e intensidad de la conducta de un sujeto en un momento determinado." (Criado del Rey Morante y Pino-Juste, 2014). Se debe entender que los motivos están compuestos por los factores que originan, dirigen y mantienen una conducta en particular, de tal manera que se considera a la motivación como un proceso complejo que integra una serie de factores que van de lo general a lo particular, y su resultado es la causa del comportamiento de un organismo o la razón por la que una entidad lleva a cabo una actividad determinada, ante la situación de excepcionalidad educativa provocada por el Covid-19 el docente debe ser soporte, promotor de la resiliencia, guía en lo académico, animador, asesor emocional y garante de la organización y coherencia institucionales (Cáceres-Muñoz, Jiménez y MartínSánchez, 2020).

De acuerdo con Vidal y García (2009), la motivación es un factor esencial y primordial en el desarrollo de cualquier actividad o trabajo a realizar. En el caso de los profesores la motivación es un factor muy importante en sus actividades cotidianas, debido a que estos se pueden estancar y tienden a bajar sus aspiraciones, o convertirse en apáticos y a establecer lo justo para pasar el día, si la motivación no es bien manejada.

De acuerdo Gadella (2020) "Durante la pandemia del Covid-19, motivar a los estudiantes en las clases virtuales ha sido crucial. Se conoce la importancia y el gran papel que juega la motivación en el aprendizaje, sin embargo, el área de la motivación del profesorado no ha recibido tanta atención". La motivación de los profesores está íntimamente relacionada a la de los estudiantes, si el profesor se siente motivado en sus clases esto dará como resultado estudiante motivados a aprender, sin embargo, una falta de motivación puede producir un efecto contrario.

Durante el confinamiento, las autoridades educativas se han preocupado mucho por los alumnos con relación a su motivación para mantener las aulas abiertas, de esta forma virtual a la que nos hemos tenido que adaptar todos, sin embargo, poco se ha hablado de la motivación de los profesores y este es un tema al que se debe prestar atención para seguir con este proyecto de educación virtual al que las circunstancias nos han orillado.

\section{PLANTEAMIETO DEL PROBLEMA}

Durante la pandemia de la Covid-19, motivar a los estudiantes en las clases virtuales ha sido crucial. Se conoce la importancia y el gran papel que juega la motivación en el aprendizaje y esto ha sido apoyado por muchas investigaciones como Beltrán et al., (2020), Gandella (2020) y Villafuerte et al., (2020). Sin embargo, el área de la motivación del profesorado no ha recibido tanta atención. Incluso los propios profesores a veces olvidan o descuidan su importancia a la hora de ejercer su profesión.

El confinamiento ha puesto a prueba todas las competencias que los profesores han adquirido a lo largo de su formación profesional, pero además han tenido que capacitarse con nuevas herramientas que les permitan mantener este nuevo sistema de escuela en casa, rompiendo con ello los viejos esquemas y paradigmas 
que han estado presentes durante más de dos siglos en los modelos educativos tradicionales hasta antes de la pandemia, para ello es necesario llevar a cabo una investigación, que permita conocer si los docentes se encuentran motivados o desmotivación en sus actividades cotidianas como profesores en la Universidad Tecnológica de Tula-Tepeji, particularmente en el Programa Educativo de Desarrollo de Negocios.

Los resultados de esta investigación nos pueden ayudar a entender las influencias negativas que afectan a los docentes diariamente y cómo estos factores pueden haber empeorado durante la pandemia al grado de provocar un cierto nivel de estrés durante el confinamiento.

\section{MÉTODO}

La metodología utilizada para la presente investigación es de tipo deductiva por medio de la estadística descriptiva a partir de los resultados del instrumento de recolección de información, mismo que se creó por medio de una plantilla de forms con 24 items, que se aplicó a una muestra representativa de 44 de los 50 profesores adscritos en el Programa Educativo Desarrollo de Negocios de la Universidad Tecnológica de Tula-Tepeji (UTTT), en el periodo comprendido de enero-abril del 2021.

Para la muestra se utilizó un nivel de confianza de 95\% y un margen de error del $5 \%$, como se muestra en la siguiente ecuación:

Ecuación para obtener la muestra

$$
n=\frac{Z^{2} N p q}{e^{2}(N-1)+Z^{2} p q}
$$

Desglose de la formula:

$\mathrm{n}=$ Numero de empresas (tamaño de la muestra)

$\mathrm{N}=$ Población (50)

$\mathrm{p}=$ Probabilidad de ocurrencia del evento $(50 \%)$

$\mathrm{q}=$ Probabilidad de que no ocurra el evento (50\%)

$\mathrm{e}=$ Error de estimación (5\%)

Z= Nivel de Confianza (1.96)

$$
n=\frac{1.96^{2}(50)(0.5)(0.5)}{(0.5)^{2}(50-1)+1.96^{2}(0.5)(0.5)}
$$

Sustituyendo los valores en la formula, el resultado para este estudio es 44 Profesores.

$$
n=44 \text { Profesores }
$$

\section{PROCEDIMIENTO}

En el estudio participaron 44 profesores del Programa Educativo Desarrollo de Negocios de los 50 adscritos para el cuatrimestre enero-abril 2021, a los cuales se les aplicó un cuestionario con 24 ítems, divididos en 4 dimensiones (ver la tabla 1), que miden aspectos como; su percepción del confinamiento a raíz de la pandemia, sus competencias digitales generales antes y durante la pandemia, nivel de motivación, por medio de una escala de Likert, y la actitud de ellos para con sus alumnos durante el confinamiento.

Tabla 1. Indicadores para la Investigación.

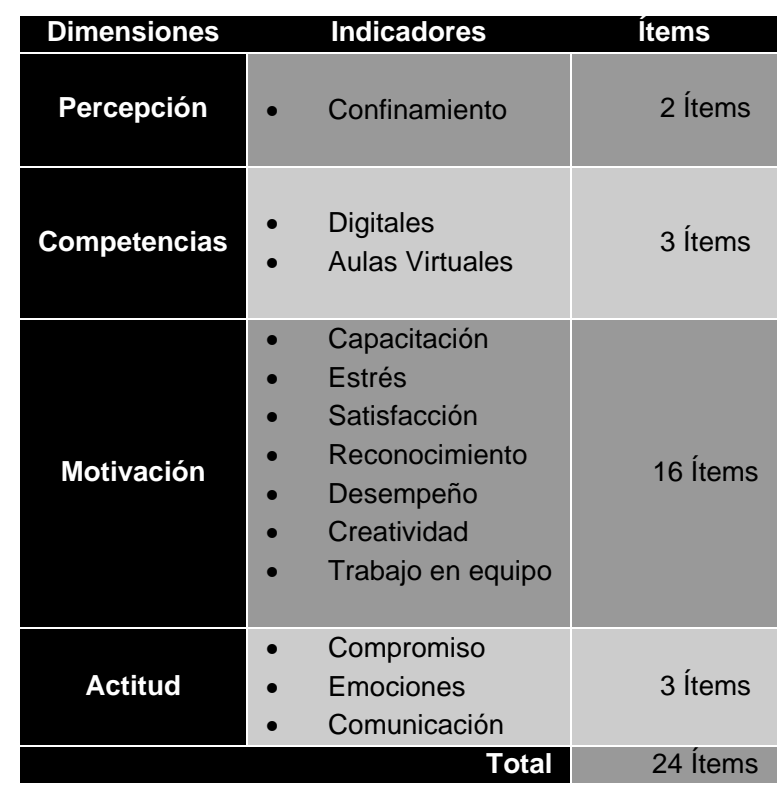

Fuente: A partir del instrumento de recolección de información.

\section{RESULTADOS}

Para el análisis de los resultados, se presentan las gráficas más representativas obtenidas en la investigación, mismas que apoyaron la identificación del nivel de motivación que estos profesores experimentan en sus actividades escolares cotidianas a partir del confinamiento por el Covid-19

Para el análisis de la $1^{\underline{a}}$ dimensión, Percepción del confinamiento, se utilizaron 2 ítems: 
De acuerso a tu criterio, LEl continamiento saritario es? 4ineouetse
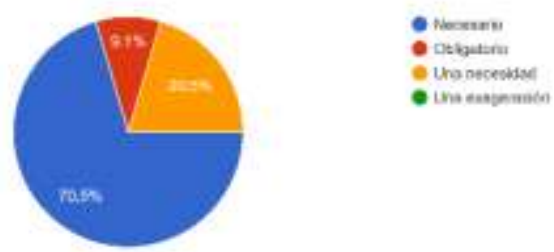

Figura 1. Necesidad Confinamiento. Fuente: Elaboración propia a partir del formulario de recolección de información.

Con relación a la necesidad del confinamiento y a un año de haberlo empezado, si se suman el $70.5 \%$ de Necesario y el $20.5 \%$ de una necesidad, tenemos que el $90 \%$ de los profesores manifiestan su aprobación al mismo, por otra parte el $9.1 \%$ restante expreso que era una obligación, sobre todo para salvaguardar la integridad de profesores, administrativos y sobre todo la de los alumnos.

En esta misma dimensión, se les pregunto a los profesores; Cuando se preveía la situación de irnos a un confinamiento por algunos meses; ¿En qué medida te sentías preparado para trabajar de manera virtual?

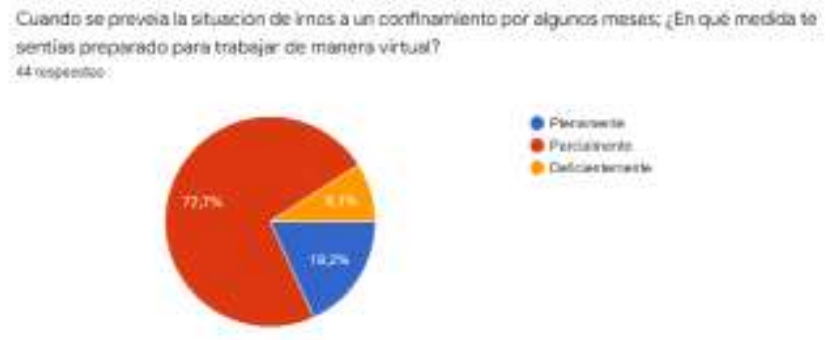

Figura 2. ¿En qué medida te sentías preparado para trabajar de manera virtual? Fuente: Elaboración propia a partir del formulario de recolección de información.

Con base en la figura 2, sólo el $18.2 \%$ se sentía plenamente preparado para enfrentar esta situación, este resultado llama mucho la atención, debido a que en la UTTT, desde el 2015 se implementó el uso de plataformas digitales como Moodle y Classroom para apoyar la formación de los alumnos, para lo cual en esa fecha ya se contaba con casi el $50 \%$ de profesores capacitados, por lo que el resultado de la investigación no concuerda, si se toma en cuenta que la mayoría de los entrevistados tienen más de 5 años trabajando en esta institución.

Para la dimensión 2, enfocada en las competencias de las TIC's se presentan los siguientes resultados.

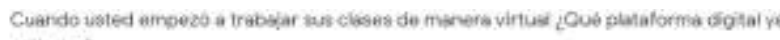
utilizan?

4tivgasatas

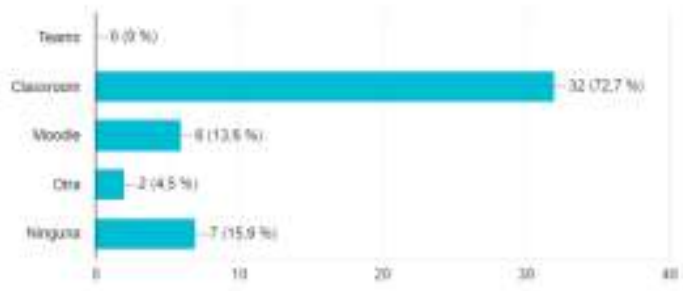

Figura 3. Aulas Virtuales, en Plataformas Digitales Utilizadas. Fuente: Elaboración propia a partir del formulario de recolección de información.

Los resultados de la 2a ${ }^{a}$. Dimensión Competencias, reafirma el supuesto presentado en la dimensión anterior debido a que las dos plataformas para aulas virtuales y clases síncronas más utilizadas son classroom y meet respectivamente con un $70 \%$ de menciones por parte de los encuestados, por encima de otra plataformas de Microsoft y Zoom, esto reafirma la labora que había venido desarrollando la UTTT en cuanto a la capacitación de la plataforma de Google como herramienta de apoyo en la formación de la comunidad universitaria.

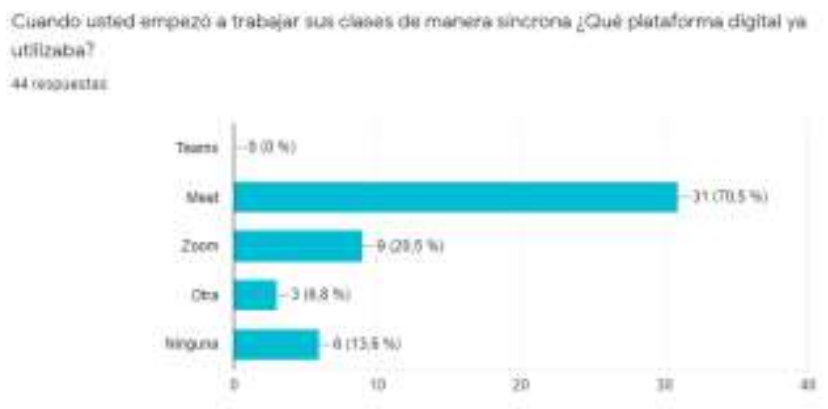

Figura 4. Plataformas para clase Síncronas. Fuente: Elaboración propia a partir del formulario de recolección de información.

Como se puede observar los profesores manejaban aulas virtuales como lo muestra la figura 3 , no así plataformas para clases síncronas como lo muestra la cuarta parte de los entrevistados, quienes indicaron no haber utilizado ninguna plataforma digital previo a la contingencia.

Afortunadamente en el último ítem de esta dimensión, los entrevistados consideraron el confinamiento, como un área de oportunidad con un $75 \%$ de menciones, esto les permitiría desarrollar sus competencias digitales para poder apoyar a los estudiantes durante esta nueva modalidad de aprendizaje. 


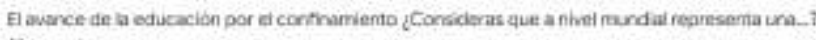
unmosata
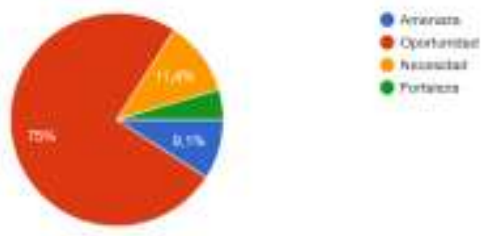

Figura 5. Perspectivas de la educación en la Contingencia. Fuente: Elaboración propia a partir del formulario de recolección de información.

Para la dimensión 3, Motivación se presentan los siguientes resultados:

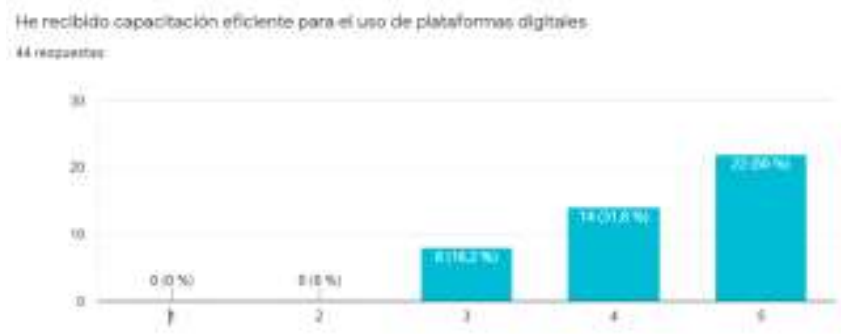

Figura 6. Capacitación. Fuente: Elaboración propia a partir del formulario de recolección de información.

De acuerdo la figura 6, los profesores han recibido suficiente capacitación para poder hacer frente a esta contingencia con el $81 \%$ de respuestas positivas.

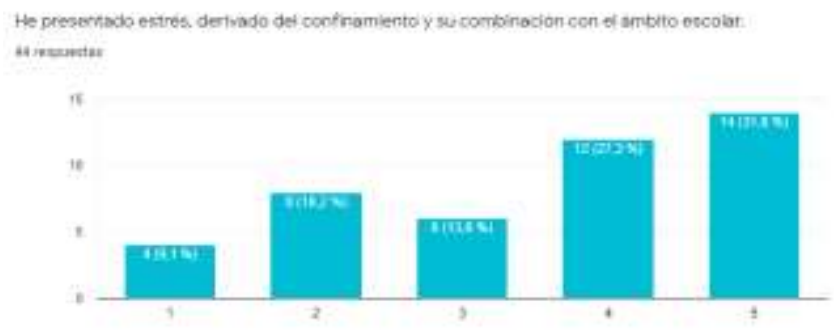

Figura 7. Síntomas de Estrés. Fuente: Elaboración propia a partir del formulario de recolección de información.

Por otra parte, el $60 \%$ manifestó haber presentado algún síntoma de estrés como lo muestra la figura 7.

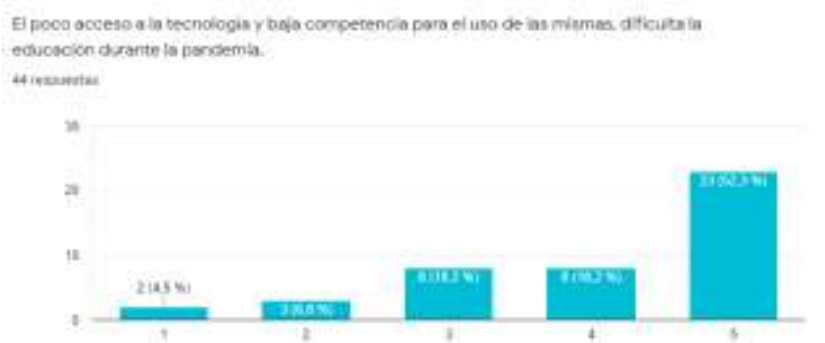

Figura 8. Problemas tecnológicos durante la pandemia. Fuente: Elaboración propia a partir del formulario de recolección de información.

El $70 \%$ de los entrevistados, manifiesta una escasa competencia por parte de los alumnos para atender las sesiones síncronas además de identificar que algunos no cuentan con las herramientas digitales que les permitan ser constantes en su formación en el contexto actual, esto se relaciona con lo manifestado por Cervantes (2020), con relación al problema de conectividad.

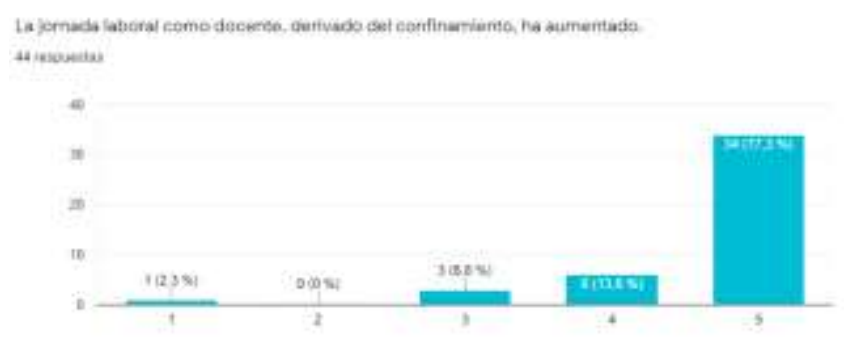

Figura 9. Jornada Laboral. Fuente: Elaboración propia a partir del formulario de recolección de información.

De acuerdo a la figura 9 , el $90 \%$ manifiesta que la jornada laboral se ha extendido a raíz de poder dar atención a todas las actividades de docencia, así como las administrativas que demandan su puesto.

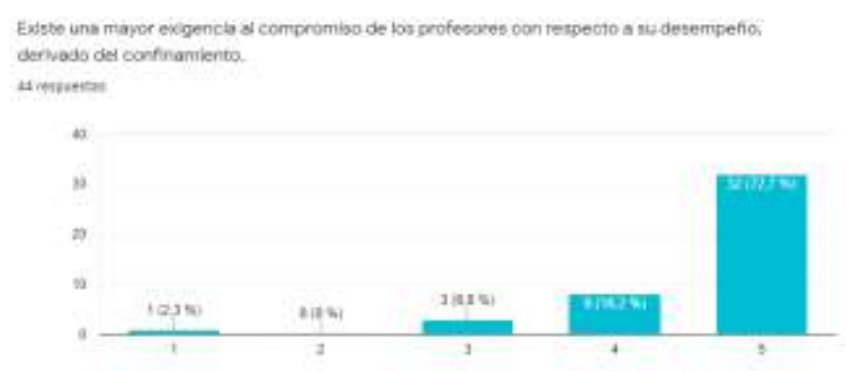

Figura 10. Compromiso. Fuente: Elaboración propia a partir del formulario de recolección de información.

El nivel de exigencia por parte de las autoridades también se ha incrementado de acuerdo a lo revisado en la figura 10 , con un $90 \%$ de las menciones, no 
obstante, los profesores reafirman su compromiso con la educación y seguir con el proyecto de escuela abierta. De acuerdo hasta lo aquí revisado, los profesores siguen con su labor académica y de acompañamiento a pesar de todas las dificultades que se han presentado en este periodo de confinamiento.

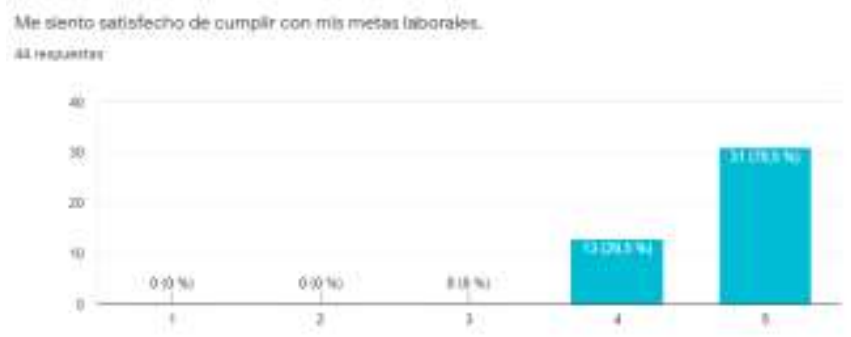

Figura 11. Satisfacción. Fuente: Elaboración propia a partir del formulario de recolección de información.

Uno de los puntos más importantes que se identificaron fue el presentado por la figura 11 , en donde el $70.5 \%$ de los entrevistados, se siente plenamente satisfecho con su labor desarrollada durante este confinamiento, mientras que el $29.5 \%$ están satisfechos, lo que da como resultado que el total de entrevistados manifiesta síntomas de satisfacción positivos de acuerdo a los datos obtenidos con el $100 \%$ de respuestas positivas.

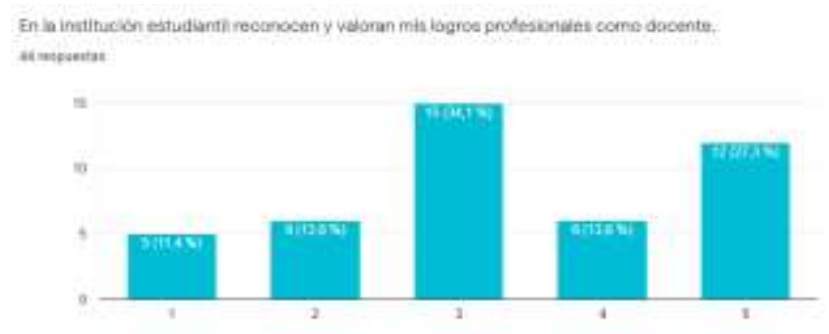

Figura 12. Reconocimiento. Fuente: Elaboración propia a partir del formulario de recolección de información.

Un indicador que también es de llamar la atención es dedicado a cuestionar, si en la institución reconocen y valoran mis logros profesionales como docente, el $60 \%$ manifestó que no se reconoce plenamente su labor docente durante el confinamiento, como se muestra en la figura 12.

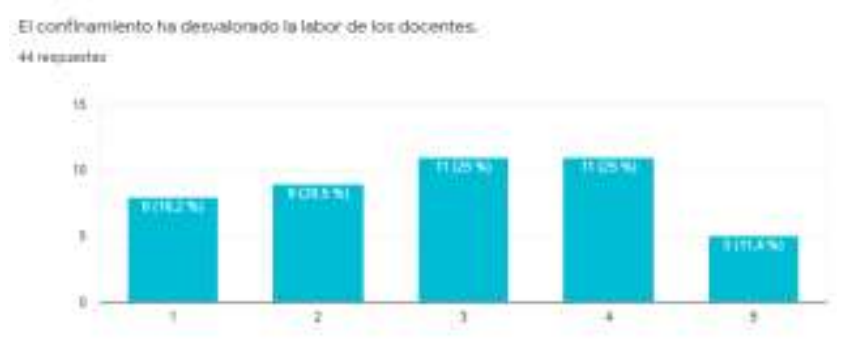

Figura 13. Desvaloración. Fuente: Elaboración propia a partir del formulario de recolección de información.

Además, como se muestra en la figura 13, los profesores se sienten desvalorados en un $70 \%$ de las menciones.

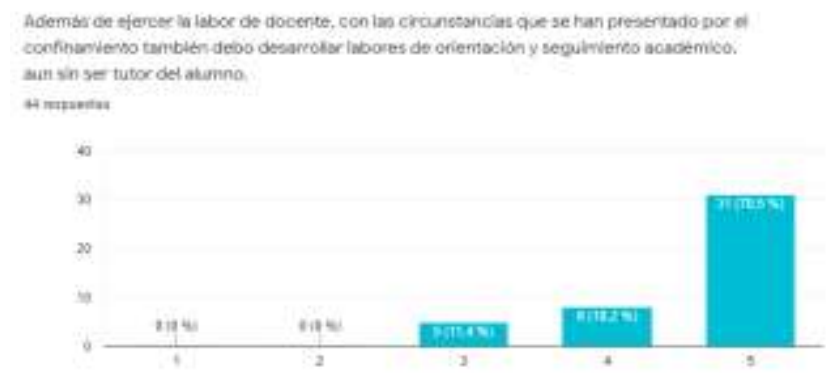

Figura 14. Tutoría Implícita. Fuente: Elaboración propia a partir del formulario de recolección de información.

Los profesores también mencionan que además de sus actividades de docencia, deben invertir parte de su tiempo en desarrollar labores de orientación y seguimiento académico, aun sin ser tutor del alumno, como se muestra en la figura 14.

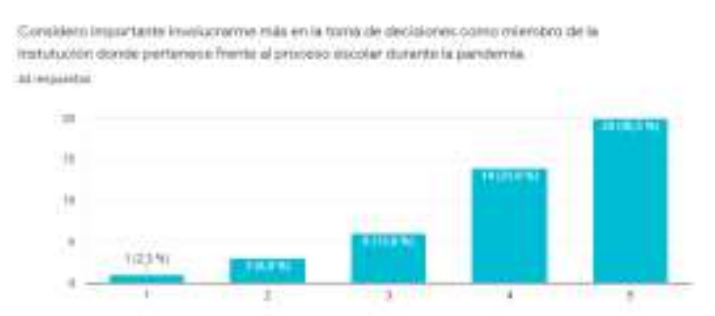

Figura 15. Toma de Decisiones. Fuente: Elaboración propia a partir del formulario de recolección de información.

En este sentido un $76 \%$ manifiesta la importancia de involucrarse más en la toma de decisiones como miembro de la institución a la que pertenece, frente al proceso escolar durante la pandemia. 


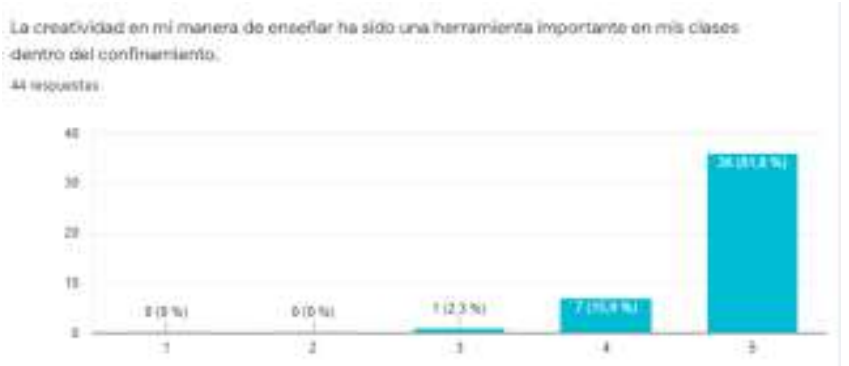

Figura 16. Creatividad. Fuente: Elaboración propia a partir del formulario de recolección de información.

De acuerdo con la figura 16, los profesores procuran desarrollar la creatividad en la preparación de sus clases, así como en la impartición de las mismas dentro del confinamiento, con un $90 \%$ de promedio de menciones en estos ítems.

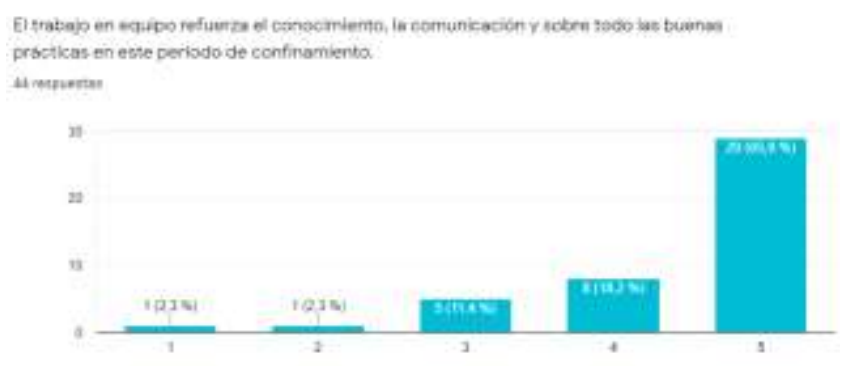

Figura 17. Comunicación. Fuente: Elaboración propia a partir del formulario de recolección de información.

Finalmente, para esta tercera dimensión, los entrevistados reconocen en el trabajo en equipo un refuerzo en el conocimiento, la comunicación y sobre todo las buenas prácticas durante esta nueva normalidad con un $85 \%$ de las menciones.

Para cerrar con la presentación de los resultados, se presenta la 4ª . Dimensión actitud.

¿Que nivei de motvacion consideras que hes puesto durante este periado de conferamiento?

44 iscpuostas
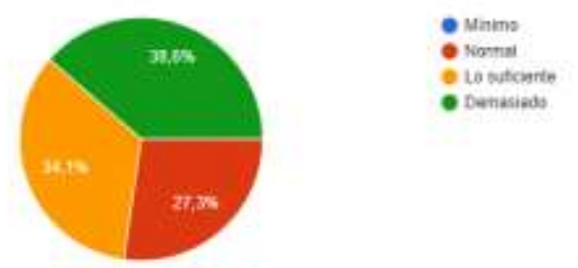

Figura 18. Nivel de Motivación. Fuente: Elaboración propia a partir del formulario de recolección de información.
El primer ítem cuestionaba, ¿Qué nivel de motivación consideras que has puesto durante este periodo de confinamiento?, las opciones de respuesta fueron a). mínimo, b). normal, c). suficiente y e). demasiado, los resultados se repartieron en los incisos b, c y e en un promedio del $30 \%$ en cada uno de los mismos. Lo cual evidencia que los profesores mantienen un buen nivel de motivación a pesar de las circunstancias que se están viviendo actualmente.

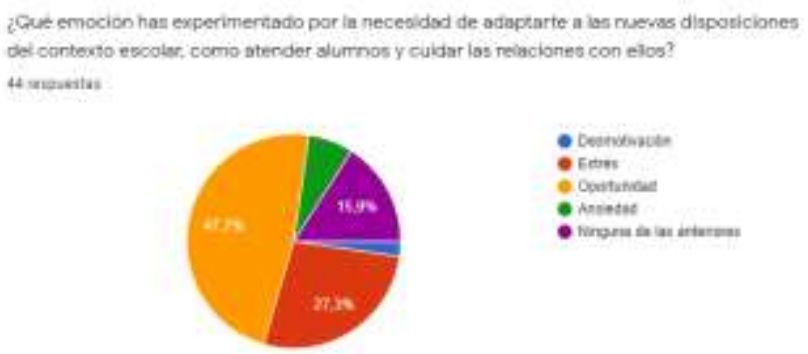

Figura 19. Adaptación. Fuente: Elaboración propia a partir del formulario de recolección de información.

Los profesores señalan que el confinamiento les ha dejado un área de oportunidad en un $50 \%$ de las menciones, aunque también el estrés es una sensación que se ha experimentado en un $30 \%$ de los profesores encuestados. Al final la adaptación la han tenido que regular de acuerdo al tiempo que siga este periodo de confinamiento.

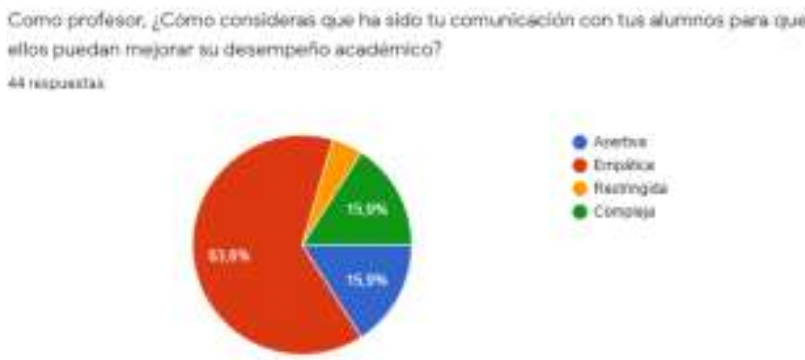

Figura 20. Comunicación. Fuente: Elaboración propia a partir del formulario de recolección de información.

Finalmente, para esta última dimensión y análisis de los resultados, los profesores mencionan que la comunicación con sus alumnos ha sido empática en un $65 \%$ de las menciones, seguidas de asertiva y compleja con un $30 \%$ en ambos casos. En términos generales los profesores han apoyado a los alumnos en estos momentos tan difíciles en donde todos deben poner lo mejor de sí para continuar con el proyecto de escuela abierta.

\section{DISCUSIÓN}

Como se mencionó anteriormente, durante la pandemia de la Covid-19, motivar a los estudiantes en las clases virtuales ha sido crucial. Sin embargo, poco se ha 
tratado sobre la motivación de los profesores, no obstante, como ha quedado demostrado en la presente investigación, los profesores han tenido que cambiar una lógica educativa de más de doscientos años, en donde se han tenido que preparar para hacer frente a una nueva realidad, con cursos y la capacitación para utilizar herramientas en muchas ocasiones desconocidas para ellos, todo para mantener el concepto de la escuela abierta, no de manera física, sino el concepto de la escuela (Escuela Musical, 2020).

A partir de emigrar de un modelo presencial a uno virtual, en esta transición los profesores han puesto mucho de su parte, capacitándose con herramientas que eran desconocidas para ellos, pero fundamentales ante esta nueva dinámica dictada por el contexto de la pandemia. Esta transición ha demandado más de su tiempo laboral que invertía tradicionalmente, con el afán de garantizar las competencias necesarias para la impartición de sus clases, pero sobre todo para asegurar formación profesional de sus alumnos.

Ante este nuevo contexto y a pesar del poco reconocimiento por parte de autoridades y de la sociedad en general, los profesores se sienten motivados para continuar con este nuevo modelo de educación a distancia que la contingencia sanitaria ha dictado a partir de la aparición del virus SRAS-CoV-2. Si bien en cierto que el panorama está lejos de cambiar, los profesores del Programa Educativo de Desarrollo de Negocios, siguen con su misión y compromiso de trabajar en aras de la juventud hidalguense y a pesar de los lapsos de estrés que ha experimentado en este confinamiento, es más el entusiasmo y compromiso que esta noble labor brinda, para quienes han dedicado su esfuerzo al engrandecimiento de Hidalgo y México.

\section{CONCLUSIONES}

Los alumnos son y serán el factor más importante en el proceso educativo, los esquemas y modelos que se presenten, deben garantizar una educación de calidad y más ahora en una situación inusual, en donde la humanidad ha tenido que confinarse para hacer frente a esta contingencia sanitaria provocada por el virus SRASCoV-2, ante esta amenaza, los maestros deben seguir siendo el pilar de donde se sustenten los cimientos que sostendrán el sistema educativo en todos los países y en particular de los alumnos del Programa Educativo de Desarrollo de Negocios de la UTTT.

Por lo tanto, es fundamental que las autoridades estén pendientes de cubrir todas las necesidades que demandan los profesores para mantenerlos, no solo preparados y capacitaos para hacer frente a esta situación, sino además motivados para continuar con la importante labor que han elegido como profesión. En la medida que un profesor se sienta pleno y motivado, transmitirá esa motivación a sus alumnos, para que, junto a los padres de familia y autoridades en general, mantengan a flote este concepto de escuela a distancia que se ha tenido que implementar a raíz de la pandemia el tiempo que sea necesario.

\section{Referencias}

[1] Beltrán, J., Venegas, M., Villar-Aguilés, A., Andrés-Cabello, S., JareñoRuiz, D. y de Gracia-Soriano, P. (2020). Educar en época de confinamiento: La tarea de renovar un mundo común. Revista de Sociología de la Educación, 13(2), 92-104. http://dx.doi.org/10.7203/RASE.13.2.17187

[2] Cáceres-Muñoz, J., Jiménez Hernández, A. S. y Martín-Sánchez, M. (2020). Cierre de Escuelas y Desigualdad Socioeducativa en Tiempos del Covid-19. Una Investigación Exploratoria en Clave Internacional. Revista Internacional de Educación para la Justicia Social, 9(3). https://doi.org/10.15366/riejs2020.9.3.011

[3] Cervantes Holguín, E. y Gutiérrez Sandoval, P. R. (2020). Resistir la Covid-19. Intersecciones en la Educación de Ciudad Juárez, México. Revista Internacional de Educación para la Justicia Social, 9(3). https://doi.org/10.15366/riejs2020.9.3.001

[4] Criado del Rey Morante, Julia y Pino-Juste, Margarita R. (2014). Estudio Sobre La Motivación del Profesorado en un Contexto Urbano. Revista Internacional de Psicología del Desarrollo y la Educación, 3 (1), 31 41. [Fecha de Consulta 17 de Agosto de 2021]. ISSN: 0214-9877. Disponible en: https://www.redalyc.org/articulo.oa?id=349851785003

[5] Escuela Musical (2020, 30 marzo). La Escuela está Abierta [Vídeo]. YouTube. https://www.youtube.com/watch?v=jR2ZjSEpmvo

[6] Gadella Kamstra, Lorena (2020) Analysis Of Efl Teachers' (De) Motivation And Awareness In Spain. (Tesis Doctoral Essex University). Repositorio Essex. http://repository.essex.ac.uk/30335/

[7] Vidal, Francisca y García, Jesús Nicasio (2009). La Motivación y El Burnout en los Profesores. Revista Internacional de Psicología del Desarrollo y la Educación, 4 (1), 161-167. [Fecha de Consulta 17 de Agosto de 2021]. ISSN: 0214-9877. Disponible en: https://www.redalyc.org/articulo.oa?id=349832323018

[8] Villafuerte, J., Bello, J., Cevallos, Y. y Bermello J. (2020). Rol de los docentes ante la crisis del Covid-19, una mirada desde el enfoque humano. REFCalE: Revista Electrónica Formación y Calidad Educativa, 8(1), 134150 . 